\title{
Bayesian nonparametric identification of Wiener systems *
}

\author{
Riccardo S. Risuleo ${ }^{\mathrm{a} F r e d r i k ~ L i n d s t e n ~}{ }^{\mathrm{b}}$ Håkan Hjalmarsson ${ }^{\mathrm{a}}$ \\ ${ }^{a}$ School of Electrical Engineering and Computer Science, KTH Royal Institute of Technology, Stockholm, Sweden \\ ${ }^{\mathrm{b}}$ Division of Statistics and Machine Learning, Linköping University, Linköping, Sweden
}

\begin{abstract}
We propose a nonparametric approach for the identification of Wiener systems. We model the impulse response of the linear block and the static nonlinearity using Gaussian processes. The hyperparameters of the Gaussian processes are estimated using an iterative algorithm based on stochastic approximation expectation maximization. In the iterations, we use elliptical slice sampling to approximate the posterior distribution of the impulse response and update the hyperparameter estimates. The same sampling is finally used to sample the posterior distribution and to compute point estimates. We compare the proposed approach with a parametric approach and a semi-parametric approach. In particular, we show that the proposed method has an advantage when a parametric model for the system is not readily available.
\end{abstract}

Key words: spline models; nonparametric estimation; system identification

\section{Introduction}

When tackling the problem of identifying a nonlinear system from data, one important challenge is the management of the tradeoff between model flexibility and risk of overfitting $([40,16])$. One solution is given by the block-oriented approach where models are compositions of simple linear and nonlinear blocks $([9])$. In this paper, we consider a particular type of block-oriented model called the Wiener model: it is a cascade composition of a linear time-invariant dynamical system followed by a memoryless nonlinear function.

The Wiener model has been used in many applications. For instance the nonlinear transformation of the output is used to model the measurements in $\mathrm{pH}$ processes $([11,22])$, polymerization reactors $([39])$, and continuously-stirred tank reactors $([14])$. Also, Wiener models and extensions have been used to model biological neurons ([5]) and muscle fibre activations ([15]). Software systems can also be studied using a combination of Wiener and other block-oriented models $([27,2])$.

\footnotetext{
* The material in this paper is an extension of results presented at the 57th IEEE Conference on Decision and Control (CDC), December 17-19, 2018, Miami, FL, USA. Corresponding author R. S. Risuleo.

Email addresses: risuleo@kth.se (Riccardo S. Risuleo), fredrik.lindsten@liu.se (Fredrik Lindsten), hjalmars@kth.se (Håkan Hjalmarsson).
}

In addition, Wiener models are used as building blocks in control applications. For instance, in Wiener nonlinear model-predictive control, basis functions are used to extend the model-predictive control approach to nonlinear systems ([18]). In Neural-network Wiener models, nonlinear systems and controllers are represented by cascades of linear dynamical systems and multilayer perceptrons $([26])$.

To identify Wiener-type cascades, many solutions have been proposed. In approaches based on maximum likelihood, a parametric model for the Wiener system is formulated and the parameters are estimated from the likelihood function using numerical optimization $([13,46])$. In particular, stochastic Wiener systems - that is, systems where the linear system has process noise- have been compared to nonlinear errors-in-variables problems ([43]) and approaches based on auxiliary models $([44])$, frequency-response functions $([42])$, and simulated maximum likelihood ([1]) have been proposed.

Other approaches based on frequency-domain measurements $([10])$, subspace approaches $([45,21,17])$, orthonormal basis functions ([41]), Bayesian methods $([30,20,47,29])$, or separate identification of the two parts of the cascade $([3,24])$ have also been proposed.

In this paper, we propose a nonparametric approach whereby both blocks of the cascade are modeled using Gaussian processes $([33,32])$. To model the linear block, 
we consider a Gaussian process model for its impulse response with a kernel that captures smoothness and exponential decay - for instance, the stable spline kernel ([31]) or some other kernel for linear system identification $([4,7])$. To model the nonlinear block, we consider a Gaussian process model for the static nonlinearity with a kernel suitable for function estimation - for instance, the squared-exponential kernel (see, [8], Chapter 2, for a reference on kernel design).

The use of nonparametric Gaussian-process models for the components of the Wiener system allows us to effectively treat identification problems where the static nonlinearity is noninvertible and the linear system is of high order. This is in contrast to other methods in the literature that restrict the class of systems considered to have invertible nonlinear blocks or parametric models of the linear block (for instance, [12], [28], and [19]).

The model we propose is an extension of the semiparametric model in [36]. There, the static nonlinearity was modeled using a combination of basis functions with unknown coefficients. In this publication, we propose a method that lets us bypass both the need to define a suitable set of basis functions and to choose an order for the combination. The resulting approach is very well suited for situations where a parametric model for the static nonlinearity is not readily available (see Section 5.2 for an example).

To estimate the hyperparameters - that is, the free parameters of the kernel functions - we follow an empirical Bayes approach and choose the hyperparameters that maximize the likelihood function after having integrated out the impulse response of the linear block and the static nonlinearity. While the marginalization with respect to the static nonlinearity admits a closed-form expression, it is not possible to explicitly integrate out the impulse response. Therefore, we propose an algorithm based on the expectation-maximization method to maximize the marginal likelihood. In particular, we use the stochastic approximation expectation maximization method [6] where the intractable expectation step is replaced by a sampling step and a stochastic approximation step. To sample from the posterior of the impulse response, we use elliptical slice sampling ([23]). This is an extension of slice sampling ([25]) designed to sample from posterior distributions given by Gaussian priors and nonlinear likelihood functions, which makes it very well suited for the problem we consider in this paper. After having found the maximum marginal likelihood estimates of the hyperparameters, we can use another sampling step to compute any point estimate from the posterior distribution of the unknowns.

\section{Problem Formulation}

In this paper, we consider systems given by the Wienertype structure presented in Figure 1.

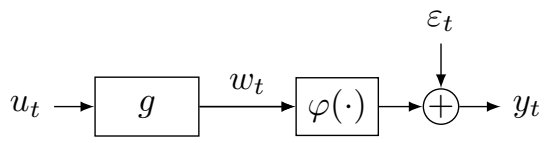

Figure 1. Block diagram of the Wiener model considered in this paper.

The structure consists of a cascade composition of a linear time-invariant dynamical system with impulse response $g$ (called linear block) and a static nonlinear function $\varphi$ (called nonlinear block). We suppose that the linear block is asymptotically stable and that there is a large enough integer $n$ such that $g_{t}$ is effectively zero for $t>n$. Here, in contrast to parametric system identification, the length $n$ of the impulse response is not chosen to tune the bias-variance tradeoff but only the computational cost of the identification algorithm and can, effectively, be chosen arbitrarily large ([32]).

We suppose that the system is excited by a known (arbitrary) input $u_{t}$. Then, the output of the linear block can be written as the discrete-time convolution

$$
w_{t}=\sum_{j=0}^{n} g_{j} u_{t-j}
$$

The output of the linear block passes through the nonlinear block and is measured with additive noise,

$$
y_{t}=\varphi\left(w_{t}\right)+\varepsilon_{t}
$$

We suppose that the measurement noise $\varepsilon_{t}$ is Gaussian and white and has variance $\beta$, which may be unknown.

For notational convenience, we use a vector notation where the $N \times 1$ vectors $y, w, \varepsilon$, and $u$ contain the samples of the corresponding signals. Similarly, the $n \times 1$ vector $g$ contains the impulse response of the linear block. With this notation, we can write the equations that describe the considered Wiener system as follows:

$$
y=\varphi(\Phi g)+\varepsilon
$$

where $\Phi$ is the $N \times n$ Toeplitz matrix given by $[\Phi]_{i, j}=$ $u_{i-j}$, which represents the vector form of the discretetime convolution (1) and where $\varphi(\cdot)$ is applied elementwise to vectors. Note that the matrix $\Phi$ contains the elements of the input $u_{t}$ for $t=-n+1, \ldots, 0$ which we assume are known. Should these initial conditions be unknown, they can be estimated with approaches similar to what was presented in [34]. 
The Wiener-system estimation problem we consider in this paper is thus as follows:

Problem 1 Given $N$ samples of the output $y$, collected from a Wiener system given by (2) in response to a known input $u$, compute the posterior distributions $\mathrm{p}(g \mid y)$ and $\mathrm{p}(\varphi \mid y)$.

From the estimated posterior distributions, we can compute point estimates such as the posterior means $\hat{g}$ and $\hat{\varphi}$, and also obtain, for instance, credible intervals for these estimates from the posterior variances.

By framing the problem in a Bayesian setting, we have replaced optimization with respect to unknown parameters with expectations computed with respect to prior distributions. In the next sections, we show how to choose suitable prior distributions and compute the expectations using Monte Carlo integration.

Remark 2 We present our results in the discrete time domain for notational convenience. However, the proposed approach can be easily implemented in continuous time using the arguments in [31]. In addition, we restrict the discussion to linear blocks of FIR or OE type; however, NARMAX or Nonlinear-BJ type models can be constructed by adding the Toeplitz matrix of the output $Y$ to the regression matrix $\bar{\Phi}=[\Phi Y]$ and considering $g$ as the stacked vector of the predictor impulse responses (see [31] for details).

Remark 3 The blocks in a Wiener system can be determined up to a scaling factor; in other words, for all real $\alpha$, the pairs $(g, x \mapsto \varphi(x))$ and $\left(\alpha g, x \mapsto \varphi\left(\frac{1}{\alpha} x\right)\right)$ describe the same input-output relation. To solve this indeterminacy, we normalize the models such that the first nonzero entry of the impulse response is positive and its $L_{2}$ gain is equal to 1. In addition, we assume that the input signal is informative enough to guarantee uniqueness in the identified model (see [13]).

\section{A new Wiener system identification method}

In this section, we present the nonparametric Bayesian model for the Wiener system that we propose. We model both the nonlinear block and the impulse response of the linear block using Gaussian processes. To find the estimates of the blocks, we follow an empirical-Bayes approach where we estimate the hyperparameters of the kernels of the Gaussian process models from the marginal likelihood function. The impulse response of the linear block together with the nonparametric model of the nonlinear block are then estimated from their posterior distributions given the data.

\subsection{Kernel-based modeling of Wiener systems}

We model the impulse response of the linear block as a realization from a zero-mean Gaussian process with hyperparameters $\rho$ and positive-definite covariance function $K(\cdot, \cdot ; \rho)$. This covariance function may be chosen among the kernels that well represent impulse responses of linear systems; see [32]. In the simulations in Section 5, we use the first-order stable-spline kernel from [31].

The chosen Gaussian-process model induces a prior distribution on the unknown impulse response vector $g$,

$$
g \sim \mathrm{p}(g ; \rho)=\mathcal{N}\left(g \mid 0, K_{\rho}\right), \quad\left[K_{\rho}\right]_{i, j}=K(i, j ; \rho) .
$$

Regarding the nonlinear block, we again use a zero-mean Gaussian-process model according to

$$
\varphi(\cdot) \sim \mathcal{G P}(0, H(\cdot, \cdot ; \eta))
$$

where the covariance function $H(\cdot, \cdot ; \eta)$ is chosen among the kernels for function estimation (for instance, radial basis functions, cubic splines, or Matern kernels; see [33]). To allow for flexible models, we let also this kernel depend on a set of hyperparameters $\eta$.

Having defined the prior densities (3) and (4), we can use (2) and the Gaussian-noise assumption on $\varepsilon$, to write out the likelihood function. To this end, we introduce an intermediate random variable $f_{t}=\varphi\left(w_{t}\right)$ that represents the Gaussian process (4) evaluated at the latent output values $w_{t}$ (see Figure 2, Left). This allows us to write the density of the data as

$$
\mathrm{p}(y \mid f ; \beta)=\mathcal{N}(y \mid f, \beta I) .
$$

From (4), the $N \times 1$ random vector $f$ is such that

$$
f \mid g ; \eta \sim \mathcal{N}\left(f \mid 0, H_{\eta}\right), \quad\left[H_{\eta}\right]_{i, j}=H\left(\Phi_{i}^{T} g, \Phi_{j}^{T} g ; \eta\right),
$$

where $\Phi_{i}^{T}$ is the $i$ th row of the regression matrix $\Phi$. Note that the prior covariance matrix $H_{\eta}$ depends on $g$; we keep this dependency implicit for notational convenience. We stress that the prior model for the static nonlinearity (4) is independent of the prior model for the impulse response (3); however, the vector of samples $f$ is given by the Gaussian process $\varphi(\cdot)$ evaluated at the locations $\Phi g$, hence its marginal distribution depends on the impulse response $g$ (see Figure 2, Middle).

As can be readily verified, $\mathrm{p}(y \mid f ; \beta)$ and $\mathrm{p}(f \mid g ; \eta)$ are conjugate Gaussian densities; this allows us to explicitly integrate out $f$ as shown in the following lemma (see Figure 2, Right).

Lemma 4 Consider the nonparametric Wiener model described by (3), (4), and (5). Then,

$$
\mathrm{p}(y \mid g ; \eta, \beta)=\mathcal{N}\left(y \mid 0, H_{\eta}+\beta I\right) .
$$



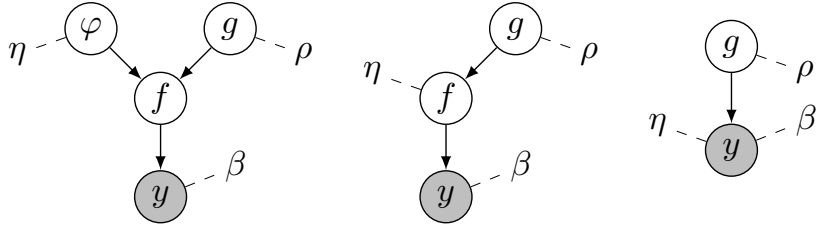

Figure 2. Bayesian networks of the proposed models. Circles represent random variables, shaded circles represent observed variables, and arrows represent conditional dependencies. Left: the complete model of the Wiener system proposed in this paper. Middle: the model after integrating out the Gaussian process $\varphi$ (note that the vector $f$ is still conditionally dependent on $g$ ). Right: Model after integrating out $f$.

In addition, let $z$ be a set of points in the domain of $\varphi(\cdot)$ and define the matrices $\left[H_{z g, \eta}\right]_{i, j}=H\left(z_{i}, \Phi_{j} g ; \eta\right)$, $\left[H_{z z, \eta}\right]_{i, j}=H\left(z_{i}, z_{j} ; \eta\right)$, then

$$
\mathrm{p}(\varphi(z) \mid y, g ; \eta, \beta)=\mathcal{N}(\varphi(z) \mid m(z, g), P(z, g))
$$

where $m(z, g)=\Gamma y, P(z, g)=H_{z z, \eta}-\Gamma H_{z g, \eta}^{T}$ and $\Gamma:=$ $H_{z g, \eta}\left(H_{\eta}+\beta I\right)^{-1}$.

\subsection{Empirical-Bayes estimation}

Using the proposed model, we can solve Problem 1 (in an empirical-Bayes sense) by estimating the posterior distribution of $g$ according to

$$
\mathrm{p}(g \mid y ; \hat{\theta}) \propto \mathrm{p}(y \mid g ; \hat{\eta}, \hat{\beta}) \mathrm{p}(g ; \hat{\rho}),
$$

where the vector of unknown hyperparameters $\hat{\theta}^{T}=$ $\left[\hat{\rho}^{T}, \hat{\eta}^{T}, \hat{\beta}\right]$ is found by maximizing the marginal likelihood $\mathrm{p}(y ; \theta)$ (also known as evidence in the machinelearning community) according to

$$
\hat{\theta}=\underset{\theta}{\arg \max } \log \mathrm{p}(y ; \theta) .
$$

Note that the size of this optimization problem is independent of the order of the system and is given by the number of hyperparameters in the model (which is typically small; for instance, in the simulations in Section 5 , we used a model with three hyperparameters).

For the Wiener model we are considering, the marginal likelihood is given by the expectation of (6) with respect to the prior of $g$,

$$
\mathrm{p}(y ; \theta)=\int \mathrm{p}(y \mid g ; \eta, \beta) \mathrm{p}(g ; \rho) \mathrm{d} g .
$$

Because of the nonlinear dependency of $y$ on the impulse response of the linear block (through the covariance matrix $H_{\eta}$ ), it is not possible to compute (9) in closed form. Therefore, to solve (8), we propose an approach based on the Expectation Maximization (EM) method (see Section 4.3).

With the estimated posterior distribution $\mathrm{p}(g \mid y ; \hat{\theta})$, we can find the posterior distribution of $\varphi$ according to

$$
\mathrm{p}(\varphi(\cdot) \mid y ; \hat{\theta})=\int \mathrm{p}(\varphi(\cdot) \mid y, g ; \hat{\eta}, \hat{\beta}) \mathrm{p}(g \mid y ; \hat{\theta}) \mathrm{d} g
$$

Because of the nonlinear dependency of $y$ on $g$, neither the density $\mathrm{p}(g \mid y ; \hat{\theta})$ nor $\mathrm{p}(\varphi(\cdot) \mid y ; \hat{\theta})$ is, in general, available in closed form. However, we can approximate $(7)$ using Markov-chain Monte Carlo (MCMC) by making a particle approximation

$$
\mathrm{p}(g \mid y ; \hat{\theta}) \approx \frac{1}{M} \sum_{i=1}^{M} \delta\left(g-\bar{g}^{(i)}\right)
$$

where $\delta(\cdot)$ is a point-mass density and where the $M$ particles $\bar{g}^{(i)}$ are drawn from $\mathrm{p}(g \mid y ; \hat{\theta})$ using MCMC. Using this particle approximation, we can compute point estimates of $g$ and other quantities of interest; in addition, we can make a Monte Carlo approximation of (10) by setting

$$
\mathrm{p}(\varphi(\cdot) \mid y ; \hat{\theta}) \approx \frac{1}{M} \sum_{i=1}^{M} \mathrm{p}\left(\varphi(\cdot) \mid y, \bar{g}^{(i)} ; \hat{\eta}, \beta\right) .
$$

Using the particle approximation, we have transformed the problem of estimating the Wiener system into the problem of sampling $\mathrm{p}(g \mid y ; \hat{\theta})$. In the next section, we show how to effectively sample this posterior distribution.

\subsection{Sampling the posterior}

As mentioned in the previous section, to estimate the Wiener system, we need to sample the posterior distribution $\mathrm{p}(g \mid y ; \hat{\theta})$. To draw the particles, note that $(7)$ is proportional to a Gaussian prior multiplied by a nonlinear likelihood function, so it can be effectively sampled using elliptical slice sampling (ESS, [23]). ESS is a specialization of the slice sampler ([25]) which, instead of using a linear slice (as in the classical slice sampling) uses an ellipse in the latent space to draw the proposed samples, with a step-size parameter that is automatically adjusted until a sample is accepted (see [23], for further details).

To generate the Markov chain, ESS starts from a state $\bar{g}^{(i)}$, a likelihood threshold $L$, and a point $\nu$ randomly drawn from the prior. Then, proposals $g^{\prime}$ are drawn from the ellipse passing through $\bar{g}^{(i)}$ and $\nu$ with an adaptively 


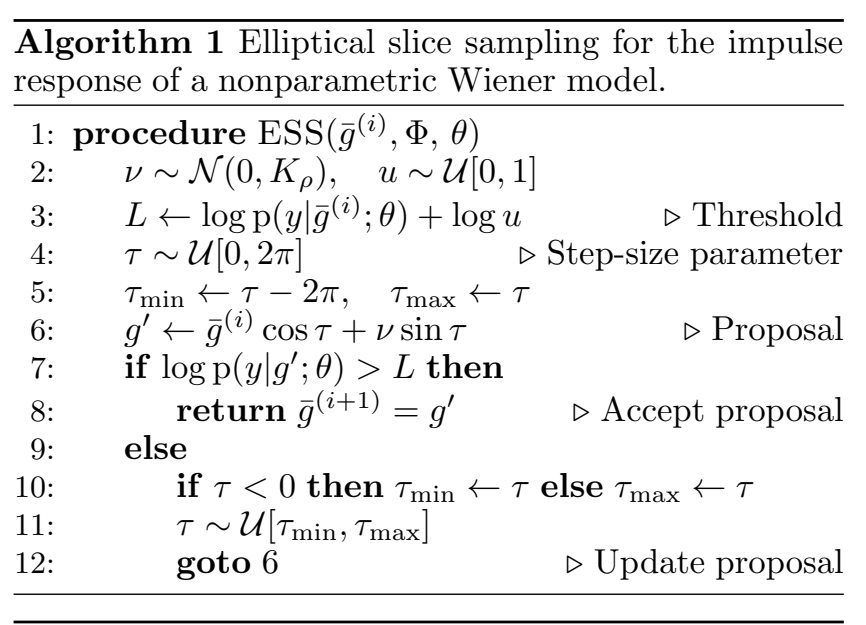

Algorithm 2 Returns $M$ samples (after a burn-in period of $B$ samples) from the posterior of $g$ for a fixed value of $\theta$.

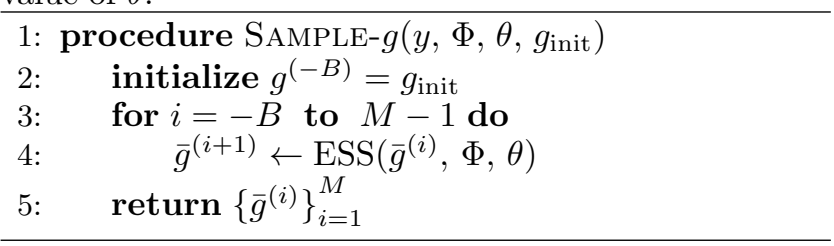

decreasing step size. Once a proposal has a likelihood that exceeds the threshold $L$, it is returned as the next state in the Markov chain. The ESS procedure is presented in Algorithm 1 (adapted from [23]). The complete MCMC algorithm to compute (11) is presented in Algorithm 2 .

In the next section, we will use the same sampler to solve the marginal likelihood problem and find the hyperparameter vector $\hat{\theta}$.

\section{Iterative hyperparameter estimation}

In this section, we propose an iterative method to estimate the hyperparameters of the nonparametric Wiener model. Because the marginal likelihood is not available, we use a variation of the EM method, the Stochastic Approximation EM (SAEM, [6]) method, to solve (8). The resulting algorithm yields simple update rules for the hyperparameters of the model of the linear block. Regarding the hyperparameters of the model of the nonlinear block, as well as the noise variance, we will propose an approximation method that results in simple update steps.

\subsection{Marginal likelihood maximization with EM}

When estimating the hyperparameters from the marginal likelihood of the proposed Wiener model, we face the difficulty that (9) is not available in closed form. However, we can interpret (8) as a maximum likelihood problem with latent variables, where the impulse response $g$ acts as the unknown latent variable to be integrated out. Because the complete-data likelihood $\mathrm{p}(y, g ; \theta)=\mathrm{p}(y \mid g ; \theta) \mathrm{p}(g ; \theta)$ is available in closed form from (3) and Lemma 4 , we can use the EM method to solve the maximum marginal likelihood problem without explicitly calculating (9).

The EM method solves a marginal likelihood problem, such as (8), iterating two steps from an arbitrary initial condition $\hat{\theta}^{(0)}$ :

(E step) Given the estimate $\hat{\theta}^{(k)}$, create a lower bound on the logarithm of (9) by computing

$$
Q\left(\theta, \hat{\theta}^{(k)}\right)=\int \log [\mathrm{p}(y, g ; \theta)] \mathrm{p}\left(g \mid y ; \hat{\theta}^{(k)}\right) \mathrm{d} g
$$

(M step) Update the parameter estimate with

$$
\hat{\theta}^{(k+1)}=\arg \max _{\theta} Q\left(\theta, \hat{\theta}^{(k)}\right) .
$$

Under some technical conditions, the sequence $\hat{\theta}^{(k)}$ converges to a local solution of the maximum marginal likelihood problem (see [48]).

Note that, for the case at hand, the E step can be split into two parts

$$
Q\left(\theta, \hat{\theta}^{(k)}\right)=Q_{1}\left(\rho, \hat{\theta}^{(k)}\right)+Q_{2}\left(\eta, \beta, \hat{\theta}^{(k)}\right)
$$

where

$$
\begin{aligned}
Q_{1}\left(\rho, \hat{\theta}^{(k)}\right) & =\int \log [\mathrm{p}(g ; \rho)] \mathrm{p}\left(g \mid y ; \hat{\theta}^{(k)}\right) \mathrm{d} g, \\
Q_{2}\left(\eta, \beta, \hat{\theta}^{(k)}\right) & =\int \log [\mathrm{p}(y \mid g ; \eta, \beta)] \mathrm{p}\left(g \mid y ; \hat{\theta}^{(k)}\right) \mathrm{d} g .
\end{aligned}
$$

So, the M step effectively decouples into the independent maximization of $Q_{1}$ and of $Q_{2}$, according to

$$
\begin{aligned}
\hat{\rho}^{(k+1)} & =\underset{\rho}{\arg \max } Q_{1}\left(\rho, \hat{\theta}^{(k)}\right), \\
\hat{\eta}^{(k+1)}, \hat{\beta}^{(k+1)} & =\underset{\eta, \beta}{\arg \max } Q_{2}\left(\eta, \beta, \hat{\theta}^{(k)}\right) .
\end{aligned}
$$

\subsection{Hyperparameters of the linear block}

Consider first the estimation of $\rho$, the hyperparameters of the linear block, in (15). At a first glance, solving (15) presents almost the same difficulties as the original problem: we do not have the posterior distribution of $g$ so we cannot compute the E step. However, this difficulty can be addressed using the Monte Carlo approximation discussed in Section 3.3. A straightforward MCMC approximation of (13) would however be very costly, since this approximation needs to be computed anew at each 
iteration of the EM algorithm. To address this issue, [6] proposed an alternative method which makes more efficient use of the MCMC samples, by reusing them across iterations in a stochastic approximation scheme. Specifically, using this method, we approximate (13) by

$$
\widehat{Q}_{1}\left(\rho, \hat{\theta}^{(k)}\right)=\left(1-\gamma_{k}\right) \widehat{Q}_{1}\left(\rho, \hat{\theta}^{(k-1)}\right)+\gamma_{k} \Delta^{(k)}(\rho)
$$

where $\gamma_{k}$ is a decreasing step-size sequence (with $\left.\sum_{k} \gamma_{k}=+\infty, \sum_{k} \gamma_{k}^{2}<+\infty\right)$ and where the stochastic update term is given by

$$
\Delta^{(k)}(\rho)=\frac{1}{M_{k}} \sum_{i=1}^{M_{k}} \log \mathrm{p}\left(\bar{g}^{(i, k)} ; \rho\right)
$$

The resulting SAEM method yields sequences of estimates that converge, again under some technical conditions, to local solutions of the marginal likelihood. Note, in particular, that SAEM converges for finite $M_{k}$ which can be chosen relatively small; see [6] for details.

The recursive form of (17) implies that the M step of the SAEM algorithm, at iteration $k$, will involve an objective function comprising $k$ terms, which means that the algorithmic complexity increases linearly in the number of steps taken. However, we can exploit the fact that the prior (3) belongs to the exponential family: this means that the M step for $\rho$ can be written in terms of a sufficient statistic. Specifically, define the posterior second moment of $g$ computed by SAEM at the $k$ th iteration as

$$
\widehat{S}^{(k)}=\left(1-\gamma_{k}\right) \widehat{S}^{(k-1)}+\frac{\gamma_{k}}{M_{k}} \sum_{i=1}^{M_{k}} \bar{g}^{(i, k)} \bar{g}^{(i, k) T} .
$$

Then, we have that the update of the hyperparameters of the linear block is as follows.

Theorem 5 The solution to $\arg \max _{\rho} \widehat{Q}_{1}\left(\rho, \hat{\theta}^{(k)}\right)$, with $\widehat{Q}_{1}\left(\rho, \hat{\theta}^{(k)}\right)$ given by $(13)$, is

$$
\hat{\rho}^{(k+1)}=\arg \max _{\rho} \operatorname{trace}\left\{K_{\rho}^{-1} \widehat{S}^{(k)}\right\}+\log \operatorname{det} K_{\rho} .
$$

\subsection{Hyperparameters of the nonlinear block}

The update of the hyperparameters of the nonlinear block $\eta$ (as well as of the noise variance $\beta$ ) is done in a similar way to how we treated $\rho$ in the preceding section. However, this update is complicated by the nonlinear dependence of $\log \mathrm{p}(y \mid g ; \eta, \beta)$ on the hyperparameters, which prevents us from transforming the stochastic approximation step into an update rule for some sufficient statistics (as we did for $\rho$ ). This implies that a stochastic approximation like (17) applied to $Q_{2}$ would yield, at the $k$ th iteration, an objective function for the M step consisting of $k$ terms, each one similar to (18).
While conceptually it is possible to keep all these terms in the objective function, we propose a more efficient method: we approximate the density $\mathrm{p}\left(g \mid y ; \hat{\theta}^{(k)}\right)$ with a point mass when computing (14) - that is, we let

$$
\mathrm{p}\left(g \mid y ; \hat{\theta}^{(k)}\right) \approx \delta\left(g-\hat{g}^{(k)}\right),
$$

where $\hat{g}^{(k)}=\left(1-\gamma_{k}\right) \hat{g}^{(k-1)}+\frac{\gamma_{k}}{M_{k}} \sum_{i=1}^{M_{k}} \bar{g}^{(i, k)}$ is the sample mean of $g \mid y ; \hat{\theta}^{(k)}$ computed by SAEM at the $k$ th iteration of the method. This implies that $Q_{2}$ is approximated as

$$
\widehat{Q}_{2}\left(\eta, \beta, \hat{\theta}^{(k)}\right)=\log \mathrm{p}\left(y \mid \hat{g}^{(k)} ; \eta, \beta\right),
$$

which allows us to replace (16) with the update

$$
\hat{\eta}^{(k+1)}, \hat{\beta}^{(k+1)}=\arg \max _{\eta, \beta} \log \mathrm{p}\left(y \mid \hat{g}^{(k)} ; \eta, \beta\right) .
$$

Using (20) in lieu of a full stochastic approximation of (14) - that is, updating the hyperparameters with (21) - has an large algorithmic advantage: directly approximating (14) using stochastic approximation would result in that the objective function for the M step at iteration $k$ is a sum of $\sum_{j=1}^{k} M_{j}$ terms. In contrast, the size and complexity of (21) are independent of the iterations and the number of particles.

Remark 6 The criterion presented in this section is similar to the maximum-likelihood inspired criterion in [35]. There, it is proposed as a model-selection criterion based on the fact that, if the true impulse response $g_{0}$ was known, the hyperparameters could be estimated from

$$
\hat{\eta}, \hat{\beta}=\underset{\eta, \beta}{\arg \max } \log \mathrm{p}\left(y \mid g_{0} ; \eta, \beta\right) .
$$

Since the impulse response is unknown, we replace it with its best estimate $\hat{g}^{(k)}$ (at the kth iteration of the EM method) and we obtain an expression that is equivalent to (21) (see [35, Section 5.3] for more details). Here, in contrast, the criterion arises from the split of the E step into (13) and (14) and from the approximation (20); however, the validity of the approach is strengthened by the positive results presented in [35].

\subsection{Wiener system identification algorithm}

The complete procedure for the identification of the nonparametric model of the Wiener structure is summarized in Algorithm 3.

After running Algorithm 3 we have the estimates of the hyperparameters of the Bayesian model of the Wiener system. Then, we can run Algorithm 2 once more (with 


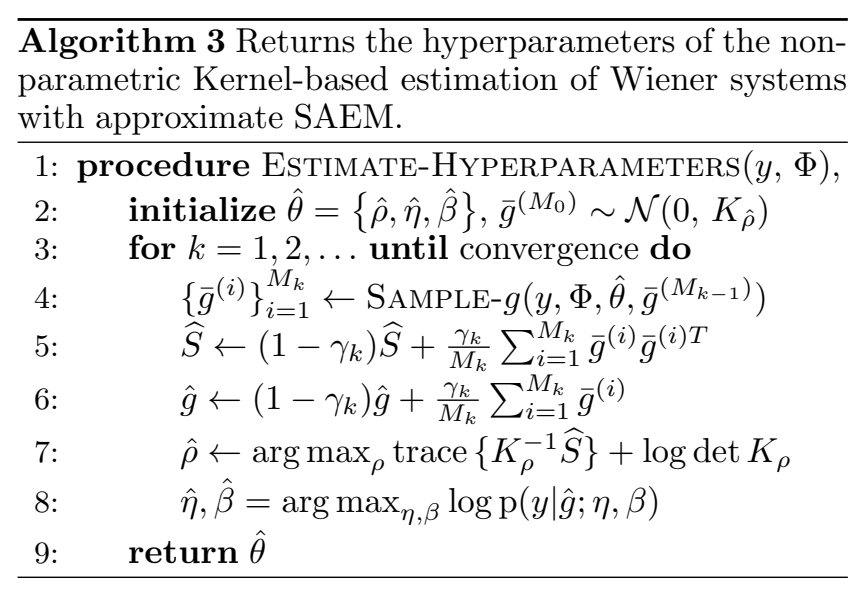

an arbitrarily large number of samples) to find the particle approximations of the posterior distributions (11) and (12) and any required point estimates: for instance, we can compute the posterior mean estimates of $g$ and $\varphi(\cdot)$ with

$$
\hat{g}=\frac{1}{M} \sum_{i=1}^{M} \hat{g}^{(i)}, \quad \hat{\varphi}(\cdot)=\frac{1}{M} \sum_{i=1}^{M} m\left(\cdot, \hat{g}^{(i)}\right),
$$

where $\hat{g}^{(i)}$ are $M$ particles drawn using Algorithm 2 .

In a straightforward implementation, the computational cost of each iteration of Algorithm 3 is of order $M_{k}\left(n^{3}+\right.$ $N^{3}+N n$ ) where the cubic terms comes from Cholesky factorizations of the covariance matrices $K_{\rho}$ and $H_{\eta}+\beta I$, respectively, and the cross-term comes from computing the matrix-vector product $w=\Phi g$. In the numerical experiments in the next section both $N$ and $n$ are sufficiently small (in the order of hundreds) so that this is not an issue. However, for large data sets it is possible to reduce the $N^{3}$ cost to $N \log N$. This is accomplished by sorting the vector $w$ and running a Kalman filter "on the components" of $w$ to efficiently compute the likelihood $\mathrm{p}(y \mid g ; \theta)$; see [37] for details.

\section{Simulations}

In the next sections, we compare the proposed estimator with alternative estimators for several different problems. In particular, we consider the estimation of Wiener systems with polynomial nonlinearities and we show that the proposed method has a performance that is comparable with state-of-the-art approaches. Then, we consider the estimation of Wiener systems with a multimodal nonlinearity that cannot easily be modeled as a polynomial.

In the simulations, we consider the following estimators:

NP (Nonparametric) The proposed nonparametric method for Wiener system identification. We model the impulse response of the linear system using the first-order stable-spline kernel ([32]),

$$
K(i, j ; \rho)=\rho^{\max (i, j)}
$$

note that there is no scaling in front of the kernel because of the nonidentifiability outlined in Remark 3 . Because of the smoothness of the static nonlinearity, we model it using a squared-exponential kernel,

$$
H(t, s ; \eta)=\eta_{1} \exp \left\{-\eta_{2}(s-t)^{2}\right\} \text {. }
$$

At each iteration, we use $M_{k}=50$ samples to compute (19); we use a burnin of $B_{1}=10$ samples for the first iteration and $B_{k}=0$ for $k>1$. The stochastic approximation rate is set to $\gamma_{k}=1 / k^{0.6}$ and the iterations are initialized with $\rho=0.5, \beta=1$, and $\eta=0.1$. The iterations are stopped once the relative change in the parameter values drops below $10^{-3}$. The posterior means (22) are computed with $M=3000$ and $B=100$.

SP (Semiparametric) The semiparametric method proposed in [36]. The impulse response is modeled using the stable-spline kernel (23) and the static nonlinearity is modeled as a combination of the first $m$ Legendre polynomials. The coefficients of the combination are estimated, together with the hyperparameters of the kernel, from the marginal likelihood of the data using SAEM with $\gamma_{k}=1 / k^{0.6}$, where $M_{k}=50$ samples are drawn after a burnin $B_{1}=10$, $B_{k}=0$ for $k>1$. After convergence, the impulse response is estimated using sampling with $M=3000$ and $B=100$. The order $m$ of the polynomial model is chosen according to a model selection criterion.

PEM (Prediction Error Method) The parametric maximum-likelihood method implemented in the Matlab system identification toolbox ([13]) with the default parameters. The orders of the transfer function and of the polynomial nonlinearity are chosen with a model-order selection criterion.

The estimators SP and PEM require the selection of a model order: in the case of SP, the number $m$ of basis functions; in the case of PEM, the number of basis functions and the order of the transfer-function model for the linear block. In the simulation, we use the following three approaches:

Or (Oracle) The order is chosen so to maximize the average fit of the estimated model to the true system (note that the oracle estimator is not usable in practice as it depends on the true system).

BIC (Bayesian Information Criterion) The model order is chosen to minimize the following information criterion $([38])$

$$
\operatorname{BIC}(p)=\frac{1}{N}\left\|\hat{\varepsilon}_{p}\right\|^{2}+\log (N) p
$$


where $\hat{\varepsilon}_{p}$ is the prediction error of the model with $p$ parameters.

CV (Cross validation) Models of different orders are estimated using the first $70 \%$ of the training data. Then, the order that minimizes the prediction error on the remaining $30 \%$ of the data is chosen and the model is re-estimated using the whole dataset to provide the final estimate.

To compare the estimators, we consider the goodnessof-fit metrics

$$
\operatorname{FIT}_{g}=1-\frac{\|g-\hat{g}\|_{2}}{\|g-\bar{g}\|_{2}}, \quad \operatorname{FIT}_{\varphi}=1-\frac{\|\varphi(z)-\hat{\varphi}(z)\|_{2}}{\|\varphi(z)-\bar{\varphi}(z)\|_{2}}
$$

Here, $\hat{g}$ is the posterior mean estimate, $g$ is the true impulse response of the system and $\bar{g}$ is the sample mean of the true impulse response; similarly, $\hat{\varphi}(z)$ is the vector of the posterior mean estimate of the static nonlinearity evaluated at a uniform grid of 200 values in $[-1,1], \varphi(z)$ is the true value of the static nonlinearity and $\bar{\varphi}(z)$ is the sample average of the true value on the grid.

\subsection{Wiener systems with polynomial nonlinearities}

In the first simulation experiment, we consider a set of 150 randomly generated Wiener systems consisting of a discrete-time transfer function followed by a polynomial nonlinearity. Each system is generated with the following procedure: First, the order $m$ of the nonlinear block is drawn uniformly at random between 3 and 10 . The nonlinear block is chosen as a finite combination of the first $m$ Legendre polynomials with coefficients drawn uniformly at random between -1 and 1 . Then, the order $d$ of the linear block is draw uniformly at random between 3 and 10. The $d$ poles are drawn in complex conjugate pairs with magnitudes uniform between 0.4 and 0.8 and the $d-1$ zeros are drawn in complex conjugate pairs with magnitudes between 0 and 0.92 . All the phases are drawn uniformly at random between 0 and $\pi / 3$. To ensure identifiability, the impulse responses are normalized to have unit $L_{2}$ gain and positive first nonzero element, and the systems are discarded until a least-squares estimation of the coefficients of the static nonlinearity from $y$ and $\Phi g$ gives positive fit (as randomly drawing both $g$ and the coefficients of the nonlinearity may lead to illconditioned identification problems).

The systems are subjected to a uniform white-noise input with amplitude 1 and the $N=500$ samples of the outputs are collected with additive Gaussian white noise with variance equal to $10 \%$ of the noiseless output variance.

The results of this simulation are presented in Figure 3. In the figure, we see the boxplots of the performance (expressed in terms of the mean of $\mathrm{FIT}_{g}$ and $\mathrm{FIT}_{\varphi}$ ) of the models estimated by the methods, for different data lengths. From the figure, it appears that the proposed nonparametric method has a performance that is comparable or better than the alternative methods. In particular, the median performance of NP is always close to the median performances of the oracle estimators (which are not usable in practice). Note that the methods SP and PEM are limited by the expressive power of the parametric part; in the next section, we present a simulation which shows that a nonparametric model of the nonlinearity can be advantageous when a parametric description of the static nonlinearity is not readily available.

\subsection{Wiener systems with sinc nonlinearities}

In the second experiment, we consider the case in which a linear-in-the-parameters model of the static nonlinearity is not easily specified. We generate a new set of 150 Wiener systems by extracting the systems from the dataset in the previous section and replacing the polynomial nonlinearities with a sinc nonlinearity

$$
\varphi(x)=\frac{\sin (6 \pi x)}{6 \pi x}
$$

We subject the systems to a uniform white-noise input with amplitude 1 and collect $N=250$ samples of the outputs with additive Gaussian white noise with variance equal to $10 \%$ of the noiseless output variance.

The results of this simulation are presented in Figure 4. In the figure, we see the median performance (expressed in terms of average fit) of the proposed nonparametric method when compared with the alternative parametric approaches. We see that the parametric approaches have lower fit over the whole interval of orders considered.

Using the proposed algorithm, implemented in Julia and run on an Intel i7-3770 CPU @ $3.40 \mathrm{GHz}$, the median time required to estimate one system in the dataset was 19.96 seconds.

\section{Conclusions}

In this paper, we have proposed a nonparametric model for Wiener system identification. We have modeled the impulse response of the linear block and the transformation of the nonlinear block as Gaussian processes. The hyperparameters of the models were estimated with stochastic approximation EM and elliptical slice sampling. We have tested the proposed approach in two simulations where we have show that the proposed approach compares favorably with state of the art methods for Wiener system identification.

One drawback of the proposed approach is its computational complexity. In particular, the method requires 


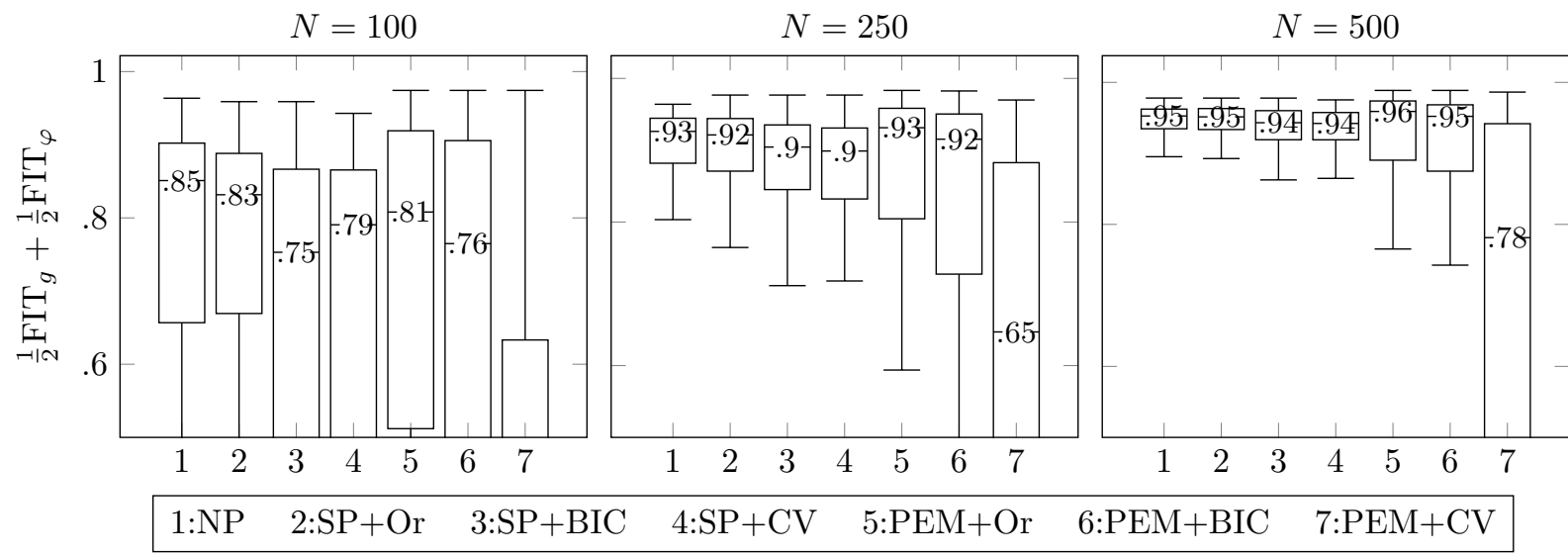

Figure 3. Boxplots of the fit of the estimated models over the 150 systems in the dataset for different data lengths $N$. The numbers indicate the median performance.

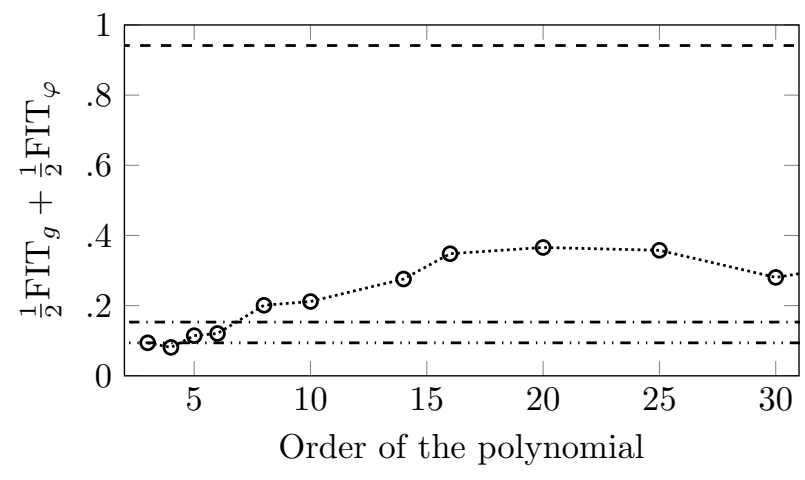

NewLEADS - New Directions in Learning Dynamical Systems (contract 2016-06079), and by the Swedish Foundation for Strategic Research via the project Probabilistic Modeling and Inference for Machine Learning (contract ICA16-0015).

\section{References}

[1] M. R. Abdalmoaty and H. Hjalmarsson. A simulated maximum likelihood method for estimation of stochastic Wiener systems. In Proc. IEEE Conf. Decis. Control (CDC), pages 3060-3065, 2016.

[2] D. Aryani, L. Wang, and T. Patikirikorala. On identification of Hammerstein and Wiener model with application to virtualised software system. Int. J. Syst. Sci., 48(6):11461161, 112016.

Figure 4. Comparsion of the median performance of the estimated nonparametric (NP) and semiparametric models (SP). We estimate semiparametric models for different orders of the static nonlinearity. We also present the median performance of the models selected by $\mathrm{BIC}$ and $\mathrm{CV}$.

the sampling of the posterior distribution of the impulse response samples at each iteration of the EM method. There are many approaches that could be followed to lower the computational burden. In particular, we are studying variational approximation methods to circumvent the need to sample at each iteration. Using variational approximation would allow us to extend the proposed nonparametric model also to HammersteinWiener and Wiener-Hammerstein cascades, and may also allow us to treat Wiener cascades with process noise.

\section{Acknowledgements}

This work was supported by the Swedish Research Council through the projects System identification: Unleashing the algorithms (contract 2015-05285), Learning of Large-Scale Probabilistic Dynamical Models (contract number: 2016-04278), and the research environment

[3] G. Bottegal, R. Castro-Garcia, and J. A. Suykens. On the identification of Wiener systems with polynomial nonlinearity. In Proc. IEEE Conf. Decis. Control (CDC), 2017.

[4] T. Chen, H. Ohlsson, and L. Ljung. On the estimation of transfer functions, regularizations and Gaussian processesRevisited. Automatica, 48(8):1525-1535, 2012.

[5] E. Chichilnisky. A simple white noise analysis of neuronal light responses. Network: Computation in Neural Systems, 12(2):199-213, 2001.

[6] B. Delyon, M. Lavielle, and E. Moulines. Convergence of a stochastic approximation version of the EM algorithm. Ann. Statist., 27(1):94-128, 31999.

[7] F. Dinuzzo. Kernels for linear time invariant system identification. SIAM J. Control Optim., 53(5):3299-3317, 2015 .

[8] D. K. Duvenaud. Automatic Model Construction with Gaussian Processes. PhD thesis, 2014.

[9] F. Giri and E. W. Bai. Block-oriented nonlinear system identification. Springer, 2010.

[10] F. Giri, Y. Rochdi, A. Radouane, A. Brouri, and F. Chaoui. Frequency identification of nonparametric Wiener systems containing backlash nonlinearities. Automatica, 49(1):124137, 2013. 
[11] J. Gomez, A. Jutan, and E. Baeyens. Wiener model identification and predictive control of a $\mathrm{pH}$ neutralisation process. IEE Proc.-Control Theory Appl., 151(3):329-338, 2004.

[12] W. Greblicki. Nonparametric identification of Wiener systems. IEEE Trans. Inf. Theory, 38(5):1487-1493, 1992.

[13] A. Hagenblad, L. Ljung, and A. Wills. Maximum likelihood identification of Wiener models. Automatica, 44(11):26972705,2008

[14] A. Harudeen and D. Banjerpongchai. Nonlinear model predictive control with Wiener model and Laguerre function for CSTR process. In Conf. Elec. Eng. Electron., Comp., Telecommun. Inf. Tec. Assoc. Thailand (ECTI). IEEE, 5 2011.

[15] I. W. Hunter and M. J. Korenberg. The identification of nonlinear biological systems: Wiener and Hammerstein cascade models. Biol. Cybern., 55(2-3):135-144, 1986.

[16] A. Juditsky, H. Hjalmarsson, A. Benveniste, B. Delyon, L. Ljung, J. Sjöberg, and Q. Zhang. Nonlinear black-box models in system identification: Mathematical foundations. Automatica, 31(12):1725-1750, 121995.

[17] S. L. Lacy and D. S. Bernstein. Identification of FIR Wiener systems with unknown, non-invertible, polynomial non-linearities. Int. J. Control, 76(15):1500-1507, 12003.

[18] M. Ławryńczuk. Nonlinear predictive control of dynamic systems represented by Wiener-Hammerstein models. Nonlinear Dyn., 86(2):1193-1214, 82016.

[19] G. Li, C. Wen, W. X. Zheng, and Y. Chen. Identification of a class of nonlinear autoregressive models with exogenous inputs based on kernel machines. IEEE Trans. Signal Process., 59(5):2146-2159, 2011.

[20] F. Lindsten, T. B. Schön, and M. I. Jordan. Bayesian semiparametric Wiener system identification. Automatica, 49(7):2053-2063, 2013

[21] M. Lovera, T. Gustafsson, and M. Verhaegen. Recursive subspace identification of linear and non-linear Wiener statespace models. Automatica, 36(11):1639-1650, 112000.

[22] S. Mahmoodi, J. Poshtan, M. R. Jahed-Motlagh, and A. Montazeri. Nonlinear model predictive control of a $\mathrm{pH}$ neutralization process based on Wiener-Laguerre model. Chem. Eng. J., 146(3):328-337, 2009

[23] I. Murray, R. Adams, and D. MacKay. Elliptical slice sampling. In Proc. Int. Conf. Artif. Intell. Stat. (AISTAT), pages 541-548, 2010.

[24] G. Mzyk and P. Wachel. Kernel-based identification of Wiener-Hammerstein system. Automatica, 83:275-281, 9 2017.

[25] R. M. Neal. Slice sampling. Ann. Statist., 31(3):705-767, 6 2003.

[26] O. Nelles. Nonlinear system identification: from classical approaches to neural networks and fuzzy models. Springer Science \& Business Media, 2013.

[27] T. Patikirikorala, L. Wang, A. Colman, and J. Han. Hammerstein-Wiener nonlinear model based predictive control for relative QoS performance and resource management of software systems. Control Eng. Pract. 20(1):49-61, 2012.

[28] M. Pawlak, Z. Hasiewicz, and P. Wachel. On nonparametric identification of Wiener systems. IEEE Trans. Signal Process., 55(2):482-492, 2007.

[29] G. Pillonetto. Consistent identification of Wiener systems: A machine learning viewpoint. Automatica, 49(9):2704-2712, 9 2013.
[30] G. Pillonetto and A. Chiuso. Gaussian processes for WienerHammerstein system identification. In Proc. IFAC Symp. System Identification (SYSID), volume 15, pages 838-843, 2009.

[31] G. Pillonetto and G. De Nicolao. A new kernel-based approach for linear system identification. Automatica, 46(1):81-93, 2010.

[32] G. Pillonetto, F. Dinuzzo, T. Chen, G. De Nicolao, and L. Ljung. Kernel methods in system identification, machine learning and function estimation: A survey. Automatica, 50(3):657-682, 2014.

[33] C. Rasmussen and C. Williams. Gaussian processes for machine learning. the MIT Press, 2006.

[34] R. S. Risuleo, G. Bottegal, and H. Hjalmarsson. On the estimation of initial conditions in kernel-based system identification. In Proc. IEEE Conf. Decis. Control (CDC), pages 1120 - 1125, 2015.

[35] R. S. Risuleo, G. Bottegal, and H. Hjalmarsson. A nonparametric kernel-based approach to hammerstein system identification. Automatica, 85:234-247, 2017.

[36] R. S. Risuleo, F. Lindsten, and H. Hjalmarsson. Semiparametric kernel-based identification of wiener systems. In Proc. IEEE Conf. Decis. Control (CDC), 2018. (to appear).

[37] S. Sarkka, A. Solin, and J. Hartikainen. Spatiotemporal learning via infinite-dimensional Bayesian filtering and smoothing: A look at Gaussian process regression through Kalman filtering. IEEE Signal Process. Mag., 30(4):51-61, 2013.

[38] G. Schwarz. Estimating the dimension of a model. Ann. Statist., 6(2):461-464, 1978.

[39] G. Shafiee, M. M. Arefi, M. R. Jahed-Motlagh, and A. A. Jalali. Nonlinear predictive control of a polymerization reactor based on piecewise linear Wiener model. Chem. Eng. J., 143(1-3):282-292, 2008.

[40] J. Sjöberg, Q. Zhang, L. Ljung, A. Benveniste, B. Delyon, P. Glorennec, H. Hjalmarsson, and A. Juditsky. Nonlinear black-box modeling in system identification: a unified overview. Automatica, 31(12):1691-1724, 1995.

[41] K. Tiels and J. Schoukens. Wiener system identification with generalized orthonormal basis functions. Automatica, 50(12):3147-3154, 2014.

[42] G. Vandersteen, Y. Rolain, and J. Schoukens. Nonparametric estimation of the frequency-response functions of the linear blocks of a Wiener-Hammerstein model. Automatica, 33(7):1351-1355, 1997.

[43] B. Wahlberg, J. Welsh, and L. Ljung. Identification of Wiener systems with process noise is a nonlinear errors-in-variables problem. In Proc. IEEE Conf. Decis. Control (CDC), pages 3328-3333, 2014.

[44] B. Wahlberg, J. Welsh, and L. Ljung. Identification of stochastic Wiener systems using indirect inference. IFACPapersOnLine, 48(28):620-625, 2015.

[45] D. Westwick and M. Verhaegen. Identifying MIMO wiener systems using subspace model identification methods. Signal Process., 52(2):235-258, 1996.

[46] A. Wills and L. Ljung. Wiener system identification using the maximum likelihood method. In Block-oriented nonlinear system identification, pages 89-110. Springer, 2010.

[47] A. Wills, T. Schön, L. Ljung, and B. Ninness. Identification of Hammerstein-Wiener models. Automatica, 49(1):70-81, 2013.

[48] C. F. J. Wu. On the convergence properties of the EM algorithm. Ann. Statist., 11(1):95-103, 1983 